\title{
Different Methods for Inducing Final Oocyte Maturation When Employing Progesterone-Primed Ovarian Stimulation Protocols
}

\section{Yen-Ju Sung}

Chang Gung Memorial Hospital at Linkou

Liang-Hsuan Chen

Chang Gung Memorial Hospital at Linkou

\section{Tzu-Hsuan Chin}

Chang Gung Memorial Hospital at Linkou

\section{Shang-Yu Huang}

Chang Gung Memorial Hospital at Linkou

\section{Hsing-Tse Yu}

Chang Gung Memorial Hospital at Linkou

\section{Chia-Lin Chang}

Chang Gung Memorial Hospital at Linkou

\section{Hong-Yuan Huang}

Chang Gung Memorial Hospital at Linkou

\section{Hsin-Shih Wang}

Chang Gung Memorial Hospital at Linkou

\section{Yung-Kuei Soong}

Chang Gung Memorial Hospital at Linkou

Hsien-Ming Wu ( $\sim$ danielwu@cgmh.org.tw)

Chang Gung Memorial Hospital at Linkou

\section{Research Article}

Keywords: progestin primed ovarian stimulation, final follicular maturation, human chorionic gonadotropin, dual trigger, embryo quality, mature oocyte

Posted Date: November 8th, 2021

DOI: https://doi.org/10.21203/rs.3.rs-1002007/v1

License: (c) (i) This work is licensed under a Creative Commons Attribution 4.0 International License. Read Full License 


\section{Abstract \\ Background}

Evidently, when undergoing $\mathrm{GnRH}$-antagonist protocols, dual trigger has proven to produce not just better quality and quantity of oocytes but also pregnancy outcome. However, not much comparative studies have been published when PPOS protocol is used for ovarian stimulation. Can the same positive outcomes be expected after the patients have been exposed to the high level of progesterone required for PPOS protocols?

\section{Methods}

In this retrospective cohort study, patients undergoing PPOS protocols were separated into three groups based on the method employed for triggering final follicular maturation, which included: (a) human chorionic gonadotropin (hCG); (b) Gonadotropin-releasing hormone-agonist (GnRH-agonist); or (c)dual trigger (GnRH-agonist $+\mathrm{hCG}$ ). Either in vitro fertilization or intracytoplasmic sperm injection (IVF/ICSI) was utilized for fertilization. Assessment comprised of their dynamic hormone profiles, embryonic analysis, and clinical outcomes.

\section{Results}

Of the 344 recruited patients, those fulfilling the Bologna criteria as poor ovarian responders and showing Estradiol (E2) $<1000 \mathrm{pg} / \mathrm{ml}$ on the day of triggering had higher oocyte maturation rate ( $82 \%$ vs $58 \%$, $\mathrm{p}<0.05)$ when triggered with dual trigger $(\mathrm{GnRH}$-agonist $+\mathrm{hCG})$ than $\mathrm{hCG}$ alone. For the patients with E2> $6500 \mathrm{pg} / \mathrm{ml}$ on the day of triggering, none of the three triggering methods demonstrated a significant advantage regarding the number of oocytes, percentage of matured oocytes, and rate of oocytes at fertilization or cleavage stages.

\section{Conclusions}

Implementing dual trigger for stimulating final follicular maturation in patients undergoing PPOS protocols is debatable. For poor ovarian response (POR) patients, dual trigger appeared to yield higher percentage of matured oocytes. In contrast, for hyper-responders, methods of triggering oocyte maturation did not affect the percentage of matured oocytes or the qualities of the embryos. For this group of patients, therefore, the agent used should be one that would reduce the risks of ovarian hyperstimulation syndrome (OHSS).

\section{Background}


Infertility afflicts about $10 \%$ of the female population, with etiologies encompassing tubal, uterine, ovulatory, or unexplained origins [1]. Whatever the cause, assisted-reproductive technology has become an essential part of the treatment, and the world's first In vitro fertilization(IVF) baby was born in 1978 [2]. More than forty years have passed since then, and with progressive maturation of techniques and continuous development of numerous pharmaceutical agents, IVF treatments have grown to become more tailored to each individual patient for optimal effects.

Suppressing the luteining hormone (LH) surge is an important part of the IVF cycle and can be clinically overcome with Gonadotropin-releasing hormone-agonist (GnRH-agonist) and Gonadotropin-releasing hormone-antagonist (GnRH-antagonist) [3]. Through downregulating the gonadotropins during the process of ovarian stimulation, $\mathrm{GnRH}$ agonist provides the additional benefit of synchronizing the size and growth of the antral follicles. On the other hand, GnRH-antagonists induce direct inhibitory effect, which has the advantages of faster onsets and less flare-ups [4].

Such downregulation is later observed in other pathways. Research has shown that progesterone secreted from the corpus luteum has the ability to inhibit the pulsatile secretion of GnRH and thus $\mathrm{LH}$, which in turn blocks the positive feedback loop of Estradiol (E2) [5]. Therefore, when high concentration of exogeneous progesterone is supplied during controlled-ovarian stimulation, LH surge can be adequately suppressed. Such usage was first documented in 2015 when medroxyprogesterone (MPA) was implemented for LH suppression [6]. Studies that followed also consistently demonstrated this effect. Thus, the term progesterone-primed ovarian stimulation protocols (PPOS) was coined [7].

However, when using PPOS protocols, all of the retrieved embryos need to be cryopreserved because fresh embryo transfer is not an option after the endometrium has been exposed to high level of progesterone required for PPOS protocol. The endometrium would have reached the receptive period too early, resulting in embryo-endometrium asynchrony [8]. Fortunately, with the significant improvement of cryopreservation, high pregnancy rate can still be achieved with thawed embryos [9]. Therefore, currently, PPOS protocols have been deemed suitable for patients seeking fertility preservation, oocyte donation, or alternative options for avoiding ovarian hyperstimulation syndrome (OHSS) [7].

During the process of ovarian stimulation, opportune triggering of final follicular maturation is a crucial step, and, previously, human chorionic gonadotropin (hCG) was used as a surrogate of LH to produce such effect. After about 30 years, $\mathrm{GnRH}$-agonists became an alternative agent for triggering final follicular maturation during GnRH-antagonist protocols with the goal of reducing risks of OHSS [10]. This concept was indeed proven by later researches, with many of them demonstrating less occurrences of OHSS with GnRH-agonist when compared with hCG; however, lower live birth and ongoing pregnancy rate (pregnancy beyond 12 weeks) and higher early miscarriage (less than 12 weeks) rate were observed with GnRH-agonist [11]. This may be attributed to defective luteal phase and decreased endometrial receptivity resulted from $\mathrm{GnRH}$-agonist trigger [12].

Hence, the concept of "dual trigger" emerged, which combined a bolus of GnRH-agonist and a bolus of hCG at the time of triggering, and has been proven advantageous. In a retrospective study, when dual 
trigger was used for normal responders, the results showed higher implantation, clinical pregnancy, and live-birth rates when compared with hCG alone [13]. Similarly, in a randomized controlled trial, when dual trigger was used for normal responders, more MIl oocytes and blastocysts were retrieved when compared with hCG trigger alone; in addition, the blastocysts obtained with dual trigger also showed higher quality [14]. In patients with diminished ovarian reserve, the use of dual trigger has also produced higher live birth rate, clinical pregnancy rate, and fertilization rate $[15,16]$. Such positive results could also be seen in patients with poor ovarian reserve, with dual trigger demonstrating higher number of oocytes and number of mature oocytes [17]. When GnRH-agonist was employed for hyper-responders, higher number of oocytes and matured oocytes were obtained when compared with triggering with hCG only. Moreover, it has the additional benefit of lowering the risks for OHSS $[18,19]$. However, not much have been published for using dual triggers for hyper-responders.

Evidently, when undergoing GnRH-antagonist protocols, dual trigger has proven to produce not just better quality and quantity of oocytes but also pregnancy outcome [14]. However, not much comparative studies have been published when PPOS protocol is used for ovarian stimulation. Can the same positive outcomes be expected after the patients have been exposed to the high level of progesterone required for PPOS protocols?

Theoretically, with high level of progesterone, FSH and LH secretions are inhibited [5]. During luteal phase stimulation, ovarian stimulation required a longer stimulation and a higher dose of total gonadotropin. These differences are not clinically significant [20]. Hence, when triggering with dual trigger or $\mathrm{GnRH}$ agonist only, the secretions of endogenous FSH and LH may also be affected. This study aims to discuss

whether different triggering methods used for final follicular maturation in PPOS protocols can affect the quality and quantity of the embryos retrieved.

\section{Materials And Methods}

\section{Study population}

This retrospective study included patients undergoing PPOS protocols in the Reproductive Center of Chang Gung Memorial Hospital (Linkou branch) from January 2017 to December 2020.

The inclusion criteria were patients enrolled for PPOS protocols with age between 20 45 years old, body mass index (BMI) less than $30 \mathrm{~kg} / \mathrm{m}^{2}$, and normal thyroid stimulating hormone (TSH) and prolactin levels. Patients with endocrine disorders, systemic diseases, or Mullerian malformations were excluded from this study.

The study was reviewed and approved by the institutional review board of the Human Investigation and Ethical Committee of Chang Gung Medical Foundation (Project no. 202100501B0; May. 4, 2021)

\section{Definition of study group}


The patients were assigned to three different final follicular maturation trigger modalities: (a) hCG (recombinant-hCG $500 \mu \mathrm{g}$; Ovidrel®; Merck Serono S.p.A.); (b) GnRH agonist (Triptorelin 0.2mg; DecapeptyI®; Ferring GmbH) (c) Dual trigger (Triptorelin 0.2mg + recombinant -hCG $500 \mu \mathrm{g}$ or $250 \mu \mathrm{g}$ )

Poor responders were defined according to the Bologna criteria [21] while high ovarian responders were patients demonstrating an E2 level greater than $6,500 \mathrm{pg} / \mathrm{mL}$ on the day of triggering.

\section{Clinical protocols}

The regime used for ovarian stimulation was tailored individually to each patient, depending on her age, $\mathrm{BMI}$, hormone levels, number of antral follicles, and previous response to stimulation. In general, on menstrual cycle day 2 or day 3 of the treatment cycles, the stimulation protocol was initiated by daily injection of recombinant-follicle-stimulating hormone (r-FSH) (Follitropin alfa; Gonal-F®, Merck Serono, SA, Geneva, Switzerland), r-FSH combined with recombinant-luteining hormone (r-LH) (Follitropin alfa + Lutropin alfa; Pergoveris $\AA$, Merck Serono, SA, Geneva, Switzerland), or human menopause gonadotrophin (HMG ; Menopur ${ }^{\circledR}$, Ferring, Kiel, Germany) at a dose of 150-225 IU/day or long-acting r-FSH 100-150 $\mu \mathrm{g}$ (Corifollitropin alfa, Elonva ${ }^{\circledR}$, Germany) in the three groups. An additional daily dose of progestin (Medroxyprogesterone 10mg once per day or Dydrogesterone $10 \mathrm{mg}$ twice per day) could be administered flexibly starting on menstrual cycle day 3 or menstrual cycle day 5 to7 when E2 was greater than 200 $\mathrm{ng} / \mathrm{mL}$ or when the leading follicle reached $10 \mathrm{~mm}$ by transvaginal ultrasonography scanning, till the day of triggering. The process for inducing final oocyte maturation would be initiated as soon as follicles were observed to be around $18 \mathrm{~mm}$ under sonography. The specific method selected would be based on patient's clinical status and the clinician's personal preference. Transvaginal retrieval of oocytes would be performed 36 hours after triggering. Based on the results of semen analysis, the matured oocytes were inseminated either by conventional insemination or intracytoplasmic sperm injection (ICSI).

Basal ovarian reserve parameters, including serum FSH, LH and E2 levels, were measured on menstrual cycle day 2 to day 3. During treatment, serum LH and E2 levels were recorded on menstrual cycle days 7 to day 9 , which corresponded to day 2 to day 4 of progestin supplementation, and the day of triggering.

\section{Primary and secondary outcome measures}

The primary endpoints of the study were number of oocytes retrieved and the proportion of matured oocytes. Secondary outcomes included fertilization rates and the percentage of embryos at the cleavage stage. Rate of matured oocyte was calculated from dividing the number of matured oocytes by the total number of oocytes retrieved. Fertilization rate was obtained from dividing the number of fertilized oocytes by the number of matured oocytes inseminated. Cleavage stage embryo was defined as an embryo presenting with two divided cells on day 2 or day 3 after inseminating. The percentage of cleavage embryo was calculated from dividing the number of cleavage stage embryos by the total number of oocytes fertilized.

\section{Statistical analysis}


All data was analyzed with Statistical Package for Social Sciences (SPSS) version 22.0 (SPSS Inc., Chicago, IL). When comparisons were made among the three groups, one-way ANOVA was utilized while Turkey HDS test was applied to identify the group causing the differences. Meanwhile, the Kruskal-Wallis test was used for intergroup comparisons of parameters without normal distributions, and the MannWhitney $U$ test was applied to identify the group causing the difference. The $X^{2}$ test was applied for comparing the qualitative data. A p value $<0.05$ was considered statistically significant.

\section{Results}

This study included a total of 344 patients undergoing PPOS protocol who were separated into three groups based on the method used for final follicular maturation: 21 patients in the hCG group, 16 patients in the $\mathrm{GnRH}$-agonist group, and 297 patients in the dual trigger ( $\mathrm{hCG}+\mathrm{GnRH}$-agonist) group. Patients' baseline characteristics, including BMI, duration of infertility, previous infertility history, antral follicle count (AFC), anti-mullerian hormone (AMH) level, and basal hormone profile, are shown in Table 1. Patients in the $\mathrm{GnRH}$-agonist group were generally younger and had significantly higher $\mathrm{AMH}$ levels.

The stimulation parameters and cycle outcomes of the three groups are presented in Table 1. Duration required for stimulation in the hCG group was longer than that of the dual trigger group $(11.9 \pm 2.93 \mathrm{vs}$ $10.02 \pm 1.71, p<0.05$ ). Meanwhile, patients in the $\mathrm{GnRH}$ agonist group had significantly higher number of follicles on day of trigger, E2 level on the day of adding progestin, E2 level on the day of triggering, number of retrieved oocytes, and number of mature oocytes when compared with the other two groups.

However, if the inclusion criteria was adjusted to only incorporate patients with $\mathrm{E} 2<1000 \mathrm{pg} / \mathrm{mL}$ on the day of triggering and classified as POR based on the Bologna criteria[21], 11 patients would be placed in the hCG group while 70 patients would be in the dual trigger group.

The baseline characteristics of this new grouping of patients are shown in Table 2. There were no significant differences between the groups. The stimulation parameters and cycle outcomes of the two groups are presented in Table 2. Patients in the dual trigger group had significantly higher percentage of oocyte maturity when compared with that of the hCG group $(0.82 \pm .26$ vs $0.58 \pm 0.50, p<0.05)$.

When analyzing the other end of the spectrum, where the inclusion criteria was changed to incorporate only patients with $\mathrm{E} 2>6500 \mathrm{pg} / \mathrm{mL}$ on the trigger day, 7 patients would be placed in the $\mathrm{GnRH}$-agonist group while 10 patients were placed in the dual trigger group. There were no significant differences, may it be the baseline characteristics, stimulation parameters, or cycle outcomes, between the two groups. These results are shown in Table 3.

\section{Discussion}

This study categorized the patients into three separate groups differentiated by the method used for final oocyte maturation. Based on their own expertise, the clinicians would choose the most optimal regime for each patient by evaluating her baseline characteristics and responses to treatment. According to their 
experiences, hCG injections would be employed for patients with low E2 levels (usually $<1500 \mathrm{pg} / \mathrm{mL}$ ) on the day of triggering. On the other hand, if patients have high E2 (usually $>3500 \mathrm{pg} / \mathrm{mL}$ ) on the day of triggering and risks for developing OHSS[22], GnRH-agonist would be used. GnRH agonist trigger for final oocyte maturation significantly reduces the risk of ovarian hyperstimulation syndrome (OHSS) in in vitro fertilization (IVF) cycles [11].

For patients falling within the middle of the spectrum, dual trigger was the main modality used, with a few exceptions that would be explained later. Therefore, upon initial assessment, it could be expected that the baseline parameters of the patients among the three groups were distinctive. However, after redefining the inclusion criteria and comparing the patients by evaluating the hCG versus dual trigger group and $\mathrm{GnRH}$ agonist versus dual trigger group, the baseline characteristics of the study population showed no statistical differences.

After eliminating the numerous confounding factors associated with different baseline characteristics, we could further analyze the data by the two new established categories: "hCG versus dual trigger" and "GnRH agonist versus dual trigger". In the "hCG versus dual trigger group", if dual trigger was prescribed for POR patients with E2 $<1000 \mathrm{pg} / \mathrm{mL}$ on the day of triggering, statistically higher rate of oocyte maturation was found. This observation was also previously described in $\mathrm{GnRH}$-antagonist cycles, where the authors believed $\mathrm{hCG}$ and $\mathrm{GnRH}$-agonist together could incite more oocyte maturation $[6,14]$. GnRHagonist has the ability to stimulate excretion of endogenous FSH and LH. LH has long been established as an important hormone for inciting final maturation of the oocytes; nonetheless, recent studies have also demonstrated the role of FSH in in vitro maturation of oocytes while animal studies have revealed the ability of FSH to induce ovulation, independently of the LH surge. It is theorized that FSH surge prompts the formation of more $\mathrm{LH}$ receptor on the luteinized granulosa cells, which then promotes the maturation of oocytes and expansion of cumulus cells [23-26].

For the "GnRH-agonist versus dual trigger group", if patients had E2>6500 pg/mL on the day of triggering, use of either method for triggering did not show any statistically differences in the outcomes. Presumably, for these hyper-responders or patients with PPOS, utilizing hCG with higher efficacy or affinity would not make a significant difference in the final outcome. Therefore, for these patients, physicians should focus on lowering the risks of developing OHSS or other complications instead. In our study, none of the patients were found to have OHSS.

The PPOS protocol used in this study included the standard method (on menstrual day 3 till day of triggering )[6] and the flexible method (on menstrual cycle day 5 to day 7 or E2>200 or follicle $>10 \mathrm{~mm}$ till day of triggering ) [27]. Current studies have not concluded on the prognostic effects of either method. A comparison of flexible PPOS with GnRH antagonist protocol in women who donated oocytes showed no significant differences in the final outcomes [27].

The choice of progestin supplementation used for our PPOS protocols included Medroxyprogesterone $10 \mathrm{mg}$ once per day and Dydrogesterone $10 \mathrm{mg}$ twice per day, with neither showing a significant advantage or disadvantage in the final outcomes in current studies [7, 28, 29]. 
With molecular structure more similar to the natural progesterone hormone, Dydrogesterone is widely used for hormone replacement, therapy, menstrual disorder treatment, endometriosis treatment, luteal support in pregnancy and threaten abortion [28]. However, use of Medroxyprogesterone is contraindicated in pregnancy [30] and breast cancer [31]. Dydrogesterone, meanwhile, appears to have fewer side effects and can be used for pregnant patients and those with history of breast cancer. However, its pricing is generally higher, and some studies have mentioned a higher rate of premature LH surge associated with its usage [29].

Researches and analysis focused on comparing live births resulted from PPOS protocols with those of $\mathrm{GnRH}$-agonist have not revealed higher rates of congenital malformation, preterm labor, low body weight, and others [32].

This is the first retrospective study discussing whether different methods for triggering follicular maturation could produce different outcomes. We hypothesized that with the higher concentration of progesterone required for PPOS cycles, it could have various effects on the agents used for triggering. Hence, different agents used for follicular maturation could possibly produce distinctive outcomes from those obtained from GnRH-antagonist cycle. Surprisingly, for POR patients, similar results were seen while not much comparisons could be made for hyper-responders due to lack of published research so far.

A major limitation to our study is the non-randomized grouping of the patients. Since all of the patients were categorized into the three study groups based on the physicians' clinical experiences, this created many differences in baseline parameters and other characteristics. For instance, most patients in the hCG group had limited number of embryos, so the retrieved follicles were all cryopreserved on Day 2 or day 3. We would not observe any blastocyst stage for that group of patients. In addition, during PPOS cycles, there were no standardization on the timing and type of medications prescribed, which could potentially affect the final results. Thirdly, due to the chemical structure of dydrogesterone, we could not accurately measure and monitor the levels of progesterone in the blood with the current diagnostic tests when using PPOS cycles [33]. Such data would have been helpful to include in the results. Lastly, longer study duration could have provided even more accurate analysis regarding the clinical pregnancy and live birth rates.

\section{Conclusion}

During PPOS cycles, if patients present with E2 $<1000 \mathrm{pg} / \mathrm{mL}$ on the day of triggering, employing dual trigger appears to have higher oocyte maturation rate $(82 \%$ vs $58 \%, p<0.05)$ when compared with that from hCG. For patients with E2 $>6500 \mathrm{pg} / \mathrm{mL}$ on the day of trigger, using dual trigger or GnRH agonist for final maturation did not exhibit significant differences in oocyte number, percentage of matured oocyte, fertilization rate, and the number of cleavage embryos. Therefore, the type of triggering agent used should be guided by aiming to lower the risks of developing OHSS. 


\section{Abbreviations}

AMH : anti-mullerian hormone ; AFC : antral follicle count BMI : body mass index E2 : Estradiol ; GnRHagonist : Gonadotropin-releasing hormone-agonist ; GnRH-antagonist : Gonadotropin-releasing hormoneantagonist ; hCG: human chorionic gonadotropin ; IVF: In vitro fertilization ; ICSI : intracytoplasmic sperm injection ; LH : luteining hormone; MPA : medroxyprogesterone ; OHSS : ovarian hyperstimulation syndrome ; PPOS: progesterone-primed ovarian stimulation protocols ; POR : poor ovarian response ; rFSH : recombinant-follicle-stimulating hormone ; r-LH : luteining hormone ; SPSS : Statistical Package for Social Sciences; TSH : thyroid stimulating hormone

\section{Declarations}

Financial Disclosure: All authors have no conflict of interest to be declared.

\section{Ethics approval and consent to participate}

This study was reviewed and approved by the institutional review board of the Human Investigation and Ethical Committee of Chang Gung Medical Foundation (Project no. 202100501B0; May. 4, 2021). Since this was a retrospective study, it had been granted an exemption from informed consent by IRB committee (review board of the Human Investigation and Ethical Committee of Chang Gung Medical Foundation). All methods were carried out in accordance with relevant guidelines and regulations of Chang Gung Medical Hospital and Taiwan.

\section{Consent for publication}

Not applicable

\section{Availability of data and materials}

The datasets used and/or analyzed during the current study are available from the corresponding author on reasonable request.

\section{Competing interest}

The authors declared that they have no competing interest

\section{Funding}

The study was supported by Chang Kung memorial hospital. No financial support was required. This study did not receive funding from any private or public sectors

\section{Authors' contribution}


H.M.W designed the study. Y.J.S and L.H.C performed the data collection and analysis. Y.J.S drafted the manuscript under the supervision of H.M.W. Y.J.S, L.H.C, T.H.C, S.Y.H, H.T.Y, C.L.C, H.Y.H, H.S.W, Y.K.S, H.M.W were involved in hypothesis generation, subjects recruitment, data management, result interpretation. All authors read and approved the final manuscript.

\section{Acknowledgements:}

Not applicable

\section{Author's information}

Department of Obstetrics and Gynecology, Chang Gung Memorial Hospital at Linkou and Chang Gung University College of Medicine, Kwei-Shan, Tao-Yuan, Taiwan.

\section{References}

1. Vander Borght, M. and C. Wyns, Fertility and infertility: Definition and epidemiology. Clinical biochemistry, 2018. 62: p. 2-10.

2. Fishel, S., First in vitro fertilization baby-this is how it happened. Fertility and sterility, 2018. 110(1): p. 5-11.

3. Macklon, N.S., et al., The science behind 25 years of ovarian stimulation for in vitro fertilization. Endocrine reviews, 2006. 27(2): p. 170-207.

4. Bosch, E., et al., Premature luteinization during gonadotropin-releasing hormone antagonist cycles and its relationship with in vitro fertilization outcome. Fertility and sterility, 2003. 80(6): p. 1444-1449.

5. Richter, T., J. Robinson, and N. Evans, Progesterone blocks the estradiol-stimulated luteinizing hormone surge by disrupting activation in response to a stimulatory estradiol signal in the ewe. Biology of reproduction, 2002. 67(1): p. 119-125.

6. Kuang, Y., et al., Medroxyprogesterone acetate is an effective oral alternative for preventing premature luteinizing hormone surges in women undergoing controlled ovarian hyperstimulation for in vitro fertilization. Fertility and sterility, 2015. 104(1): p. 62-70. e3.

7. Ata, B., et al., Progestins for pituitary suppression during ovarian stimulation for ART: a comprehensive and systematic review including meta-analyses. Human Reproduction Update, 2021. 27(1): p. 48-66.

8. Massin, N., New stimulation regimens: endogenous and exogenous progesterone use to block the $L H$ surge during ovarian stimulation for IVF. Human reproduction update, 2017. 23(2): p. 211-220.

9. Nagy, Z.P., D. Shapiro, and C.-C. Chang, Vitrification of the human embryo: a more efficient and safer in vitro fertilization treatment. Fertility and sterility, 2020. 113(2): p. 241-247.

10. GONEN, Y., et al., Use of gonadotropin-releasing hormone agonist to trigger follicular maturation for in vitro fertilization. The Journal of Clinical Endocrinology \& Metabolism, 1990. 71(4): p. 918-922. 
11. Youssef, M.A., et al., Gonadotropin-releasing hormone agonist versus HCG for oocyte triggering in antagonist assisted reproductive technology cycles. Cochrane Database of Systematic Reviews, 2011(1).

12. Engmann, L. and C. Benadiva. Ovarian hyperstimulation syndrome prevention strategies: luteal support strategies to optimize pregnancy success in cycles with gonadotropin-releasing hormone agonist ovulatory trigger. in Seminars in reproductive medicine. 2010. () Thieme Medical Publishers.

13. Lin, M.-H., et al., Dual trigger with combination of gonadotropin-releasing hormone agonist and human chorionic gonadotropin significantly improves the live-birth rate for normal responders in GnRH-antagonist cycles. Fertility and sterility, 2013. 100(5): p. 1296-1302.

14. Haas, J., et al., GnRH agonist and hCG (dual trigger) versus hCG trigger for final follicular maturation: a double-blinded, randomized controlled study. Human Reproduction, 2020. 35(7): p. 1648-1654.

15. Lin, M.-H., et al., Dual trigger with gonadotropin releasing hormone agonist and human chorionic gonadotropin significantly improves live birth rate for women with diminished ovarian reserve. Reproductive Biology and Endocrinology, 2019. 17(1): p. 1-7.

16. Chern, C.-U., et al., Dual-trigger improves the outcomes of in vitro fertilization cycles in older patients with diminished ovarian reserve: A retrospective cohort study. Plos one, 2020. 15(7): p. e0235707.

17. Zhang, J., et al., Dual trigger of final oocyte maturation in poor ovarian responders undergoing IVF/ICSI cycles. Reproductive biomedicine online, 2017. 35(6): p. 701-707.

18. Tannus, S., et al., Reproductive outcomes after a single dose of gonadotropin-releasing hormone agonist compared with human chorionic gonadotropin for the induction of final oocyte maturation in hyper-responder women aged 35-40 years. Fertility and sterility, 2017. 107(6): p. 1323-1328. e2.

19. Tan, J., et al., GnRH triggering may improve euploidy and live birth rate in hyper-responders: a retrospective cohort study. Journal of Assisted Reproduction and Genetics, 2020. 37(8): p. 19391948.

20. Boots, C., et al., Ovarian stimulation in the luteal phase: systematic review and meta-analysis. Journal of assisted reproduction and genetics, 2016. 33(8): p. 971-980.

21. Ferraretti, A., et al., ESHRE consensus on the definition of 'poor response'to ovarian stimulation for in vitro fertilization: the Bologna criteria. Human reproduction, 2011. 26(7): p. 1616-1624.

22. D'Angelo, A., et al., Value of the serum estradiol level for preventing ovarian hyperstimulation syndrome: a retrospective case control study. Fertility and sterility, 2004. 81(2): p. 332-336.

23. Zelinski-Wooten, M., et al., Endocrinology: Follicle stimulating hormone alone supports follicle growth and oocyte development in gonadotrophin-releasing hormone antagonist-treated monkeys. Human Reproduction, 1995. 10(7): p. 1658-1666.

24. Andersen, C.Y., et al., FSH-induced resumption of meiosis in mouse oocytes: effect of different isoforms. Molecular Human Reproduction, 1999. 5(8): p. 726-731.

25. Strickland, S. and W. Beers, Studies on the role of plasminogen activator in ovulation. In vitro response of granulosa cells to gonadotropins, cyclic nucleotides, and prostaglandins. Journal of Biological Chemistry, 1976. 251(18): p. 5694-5702. 
26. Eppig, J.J., FSH stimulates hyaluronic acid synthesis by oocyte-cumulus cell complexes from mouse preovulatory follicles. Nature, 1979. 281(5731): p. 483-484.

27. Yildiz, S., et al., Comparison of a novel flexible progestin primed ovarian stimulation protocol and the flexible gonadotropin-releasing hormone antagonist protocol for assisted reproductive technology. Fertility and sterility, 2019. 112(4): p. 677-683.

28. Yu, S., et al., New application of dydrogesterone as a part of a progestin-primed ovarian stimulation protocol for IVF: a randomized controlled trial including 516 first IVF/ICSI cycles. Human Reproduction, 2018. 33(2): p. 229-237.

29. Huang, J., et al., Progestin-primed ovarian stimulation with dydrogesterone versus medroxyprogesterone acetate in women with polycystic ovarian syndrome for in vitro fertilization: a retrospective cohort study. Drug design, development and therapy, 2019. 13: p. 4461.

30. Katz, Z., et al., Teratogenicity of progestogens given during the first trimester of pregnancy. Obstetrics and gynecology, 1985. 65(6): p. 775-780.

31. Ruan, X. and A.O. Mueck, The choice of progestogen for HRT in menopausal women: breast cancer risk is a major issue. Hormone Molecular Biology and Clinical Investigation, 2019. 37(1).

32. Zolfaroli, l., et al., Impact of progestin ovarian stimulation on newborn outcomes: a meta-analysis. Journal of assisted reproduction and genetics, 2020: p. 1-10.

33. Abdel-Hamid, M., et al., Determination of dydrogesterone in human plasma by tandem mass spectrometry: Application to therapeutic drug monitoring of dydrogesterone in gynecological disorders. Chromatographia, 2006. 64(5): p. 287-292.

\section{Tables}

Table 1. IVF cycle characteristics, endocrine parameters, and outcomes in different study groups 


\begin{tabular}{|c|c|c|c|c|}
\hline & hCG & GnRH-agonist & Dual trigger ${ }^{a}$ & $\stackrel{p}{\text { Value }}$ \\
\hline No of patients & 21 & 16 & 297 & \\
\hline Age (years) & $37.52 \pm 5.58$ & $33.56 \pm 3.91$ & $37.64 \pm 4.76$ & $<0.05^{\star}$ \\
\hline BMI $\left(\mathrm{kg} / \mathrm{m}^{2}\right)$ & $24.02 \pm 5.32$ & $22.37 \pm 3.24$ & $22.48 \pm 3.63$ & 0.187 \\
\hline $\mathrm{AMH}(\mathrm{ng} / \mathrm{ml})$ & $2.29 \pm 2.51$ & $8.12 \pm 4.00$ & $2.74 \pm 2.66$ & $<0.05^{\star}$ \\
\hline Infertility years & $3.73 \pm 3.41$ & $3.73 \pm 2.78$ & $3.83 \pm 3.15$ & 0.987 \\
\hline Primary infertility & $65 \%(13 / 20)$ & $81 \%(13 / 16)$ & $61 \%(177 / 288)$ & \\
\hline Male factor & $12 \%(2 / 17)$ & $40 \%(6 / 15)$ & $13 \%(36 / 280)$ & \\
\hline Basal FSH(IU/L) & $7.99 \pm 4.79$ & $6.25 \pm 2.24$ & $9.22 \pm 7.48$ & 0.227 \\
\hline Basal LH(IU/L) & $4.99 \pm 3.40$ & $6.32 \pm 2.61$ & $4.79 \pm 3.92$ & 0.297 \\
\hline Basal E2(pg/ml) & $46.09 \pm 46.20$ & $31.19 \pm 13.29$ & $45.52 \pm 76.64$ & 0.761 \\
\hline Day of stimulation & $11.19 \pm 2.93$ & $10.63 \pm 0.96$ & $10.02 \pm 1.71$ & $<0.05^{\star}$ \\
\hline $\begin{array}{l}\text { Total follicles on day of } \\
\text { triggering }\end{array}$ & $6.52 \pm 5.05$ & $14.88 \pm 3.63$ & $8.10 \pm 4.48$ & $<0.05^{\star}$ \\
\hline $\begin{array}{l}\text { E2 on the day of progestin } \\
(\mathrm{pg} / \mathrm{ml})\end{array}$ & $675.36 \pm 691.33$ & $\begin{array}{l}2079.57 \pm \\
1215.99\end{array}$ & $672.93 \pm 803.54$ & $<0.05^{\star}$ \\
\hline $\begin{array}{l}\text { LH on the day of progestin } \\
(\mathrm{IU} / \mathrm{L})\end{array}$ & $5.26 \pm 3.83$ & $8.18 \pm 6.55$ & $5.39 \pm 5.09$ & 0.101 \\
\hline $\begin{array}{l}\text { E2 on the day of triggering } \\
(\mathrm{pg} / \mathrm{ml})\end{array}$ & $\begin{array}{l}1282.19 \pm \\
1481.59\end{array}$ & $\begin{array}{l}6180.63 \pm \\
3338.36\end{array}$ & $\begin{array}{l}1944.61 \pm \\
1892.78\end{array}$ & $<0.05^{\star}$ \\
\hline $\begin{array}{l}\text { LH on the day of triggering } \\
(\mathrm{IU} / \mathrm{L})\end{array}$ & $4.34 \pm 4.55$ & $3.42 \pm 2.08$ & $4.52 \pm 4.53$ & 0.624 \\
\hline Retrieved oocytes & $8.48 \pm 10.42$ & $27.38 \pm 10.95$ & $11.23 \pm 10.68$ & $<0.05^{\star}$ \\
\hline Mature oocytes & $7.55 \pm 9.82$ & $21.00 \pm 8.43$ & $9.02 \pm 9.34$ & $<0.05^{\star}$ \\
\hline Oocyte maturity rate & $0.72 \pm 0.36$ & $0.79 \pm 0.20$ & $0.80 \pm 0.20$ & 0.399 \\
\hline No of $2 \mathrm{PN}$ & $4.29 \pm 6.73$ & $16.00 \pm 6.61$ & $6.43 \pm 6.63$ & $<0.05^{\star}$ \\
\hline No of blastocyst & $2 \pm 4.57$ & $9.87 \pm 5.10$ & $3.13 \pm 4.11$ & $<0.05^{\star}$ \\
\hline
\end{tabular}

Data are expressed in mean \pm SD or frequency $(\%)$

$\mathrm{BMI}=$ body mass index; $\mathrm{AMH}=$ anti-Müllerian hormone; $\mathrm{ICSI}=$ intracytoplasmic sperm injection

$\mathrm{FSH}=$ follicle stimulating hormone; $\mathrm{LH}=$ luteining hormone; $\mathrm{E} 2=$ estradiol; $2 \mathrm{PN}=2$-pronuclear zygote 
Dual trigger $^{\mathrm{a}}: \mathrm{GnRH}$-agonist $+\mathrm{hCG}$

Watured oocytes were inseminated either by conventional insemination or ICSI

Table 2. POR patient characteristics, endocrine parameters, and outcomes in different study groups

\begin{tabular}{|llll|}
\hline & hCG & Dual trigger & p Value \\
\hline No of patients & 11 & 70 & \\
\hline Age (years) & $40.73 \pm 4.74$ & $40.61 \pm 3.90$ & 0.269 \\
\hline BMI (kg/m $\left.{ }^{2}\right)$ & $25.97 \pm 6.39$ & $23.54 \pm 4.16$ & 0.102 \\
\hline AMH (ng/ml) & $0.71 \pm 0.40$ & $0.62 \pm 0.49$ & 0.591 \\
\hline Infertility years & $4.27 \pm 3.88$ & $4.42 \pm 3.66$ & 0.865 \\
\hline Primary infertility & $58 \%(7 / 12)$ & $64 \%(76 / 119)$ & \\
\hline Male factor & $20 \%(2 / 10)$ & $11 \%(12 / 106)$ & \\
\hline Basal FSH (IU/L) & $9.96 \pm 5.59$ & $13.30 \pm 12.10$ & 0.574 \\
\hline Basal LH (IU/L) & $4.58 \pm 4.46$ & $4.62 \pm 3.52$ & 0.579 \\
\hline Basal E2 (pg/ml) & $34.79 \pm 30.86$ & $44.02 \pm 48.01$ & 0.773 \\
\hline Days of stimulation & $12.45 \pm 3.20$ & $9.73 \pm 2.16$ & 0.110 \\
\hline Total follicles on day of triggering & $2.55 \pm 1.29$ & $3.54 \pm 1.85$ & 0.169 \\
\hline E2 on the day of progestin $(\mathrm{pg} / \mathrm{ml})$ & $180.75 \pm 214.56$ & $203.01 \pm 181.84$ & 0.692 \\
\hline LH I on the day of progestin (IU/L) & $6.54 \pm 5.11$ & $7.25 \pm 7.20$ & 0.806 \\
\hline E2 on the day of triggering (pg/ml) & $384.73 \pm 191.85$ & $391.47 \pm 243.48$ & 0.143 \\
\hline LH on the day of triggering (IU/L) & $6.09 \pm 6.01$ & $7.64 \pm 7.36$ & 0.884 \\
\hline Retrieved oocytes & $1.36 \pm 1.50$ & $1.97 \pm 1.20$ & 0.619 \\
\hline Mature oocytes & $1.00 \pm 1.55$ & $1.56 \pm 1.07$ & 0.313 \\
\hline Oocyte maturity rate & $0.58 \pm 0.50 \%$ & $0.82 \pm 0.26 \%$ & $P<0.05 *$ \\
\hline No of 2PN by ICSI & $0.9 \pm 1.60$ & $1.15 \pm 1.06$ & 0.594 \\
\hline Fertilization rate by ICSI & $0.88 \pm 0.25 \%$ & $0.71 \pm 0.42 \%$ & 0.954 \\
\hline Cleavage stage rate & $1 \pm 0 \%$ & $0.94 \pm 0.22 \%$ & 0.354 \\
\hline No of blastocyst & $0 \pm 0$ & $0.20 \pm 0.60$ & P<0.05* \\
\hline
\end{tabular}


Data are expressed in mean \pm SD or frequency $(\%)$

$\mathrm{BMI}=$ body mass index; $\mathrm{AMH}=$ anti-Müllerian hormone; $\mathrm{ICSI}=$ intracytoplasmic sperm injection

$\mathrm{FSH}=$ follicle stimulating hormone; $\mathrm{LH}=$ luteining hormone; $\mathrm{E} 2=$ estradiol; 2PN= 2-pronuclear zygote

Dual trigger ${ }^{\mathrm{a}}: \mathrm{GnRH}$-agonist $+\mathrm{hCG}$

Table 3. Hyper-responder patient characteristics, endocrine parameters, and outcomes in different study groups 


\begin{tabular}{|llll|}
\hline & GnRH-agonist & Dual trigger & p Value \\
\hline No of patients & 7 & 10 & \\
\hline Age (years) & $34.14 \pm 4.41$ & $34.30 \pm 4.19$ & 0.718 \\
\hline BMI (kg/m $\left.{ }^{2}\right)$ & $21.26 \pm 3.03$ & $21.73 \pm 3.27$ & 0.761 \\
\hline AMH (ng/ml) & $6.47 \pm 3.23$ & $8.13 \pm 3.09$ & 0.236 \\
\hline Infertility years & $3.57 \pm 3.46$ & $2.60 \pm 0.97$ & 0.087 \\
\hline Primary infertility & $86 \%(6 / 7)$ & $60 \%(6 / 10)$ & \\
\hline Male factor & $43 \%(3 / 7)$ & $10 \%(1 / 10)$ & \\
\hline Basal FSH (IU/L) & $5.64 \pm 2.20$ & $6.21 \pm 1.72$ & 0.525 \\
\hline Basal LH (IU/L) & $6.34 \pm 4.00$ & $7.26 \pm 5.46$ & 0.642 \\
\hline Basal E2 (pg/ml) & $35.96 \pm 11.38$ & $42.89 \pm 21.82$ & 0.066 \\
\hline Days of stimulation & $10.86 \pm 0.69$ & $10.60 \pm 1.43$ & 0.215 \\
\hline Total follicle on day of triggering & $14.71 \pm 3.15$ & $11.70 \pm 3.06$ & 0.969 \\
\hline E2 on the day of progestin $(\mathrm{pg} / \mathrm{ml})$ & $2336.86 \pm 754.29$ & $3275.20 \pm 1497.92$ & 0.226 \\
\hline LH on the day of progestin $(\mathrm{IU} / \mathrm{L})$ & $10.64 \pm 7.56$ & $7.87 \pm 9.43$ & 0.893 \\
\hline E2 on the day of triggering $(\mathrm{pg} / \mathrm{ml})$ & $9043.43 \pm 2558.62$ & $8651.20 \pm 1992.62$ & 0.634 \\
\hline LH on the day of triggering (IU/L) & $3.81 \pm 1.35$ & $3.81 \pm 2.68$ & 0.055 \\
\hline Retrieved oocytes & $28.00 \pm 11.66$ & $36.40 \pm 15.62$ & 0.188 \\
\hline Mature oocytes & $22.71 \pm 10.42$ & $30.70 \pm 16.49$ & 0.187 \\
\hline Oocyte maturity rate & $0.82 \pm 0.20$ & $0.83 \pm 0.17$ & 0.655 \\
\hline No of 2PN by ICSI & $15.29 \pm 7.25$ & $19.9 \pm 9.16$ & 0.594 \\
\hline Fertilization rate by ICSI & $0.73 \pm 0.17$ & $0.71 \pm 0.20$ & 0.702 \\
\hline Cleavage stage rate & $0.93 \pm 0.12$ & $0.98 \pm 0.04$ & 0.164 \\
\hline No of blastocyst & $9.43 \pm 4.47$ & $10.80 \pm 4.57$ & 0.674 \\
\hline
\end{tabular}

Data are expressed in mean \pm SD or frequency $(\%)$

$\mathrm{BMI}=$ body mass index; $\mathrm{AMH}=$ anti-Müllerian hormone; $\mathrm{ICSI}=$ intracytoplasmic sperm injection

$\mathrm{FSH}=$ follicle stimulating hormone; $\mathrm{LH}=$ luteining hormone; $\mathrm{E} 2=$ estradiol; $2 \mathrm{PN}=2$-pronuclear zygote Dual trigger ${ }^{\mathrm{a}}$ : GnRH-agonist + hCG 